\title{
EDITORIAL
}

\section{In the December, 2010 issue of CLINICS}

\author{
Mauricio Rocha e Silva
}

As CLINICS completes its $6^{\text {th }}$ year of existence we believe we have reasons for a little bit of celebration. In June 2010 The Journal of Citation Reports of the Institute for Scientific Information posted its first Impact Factor for CLINICS, which confirmed the positive trend we had been monitoring through the Scimago Index of Scopus. Over the years 2006 2008 we had consistently maintained a cites per document level ranging between 1.0 and 1.3. In June, the 2009 Impact Factor came out at 1.591, the second highest Brazilian IF.

We also decided to give the journal a face lift and as from August 2010 we are posting an e-book of each issue in our internet site. In the near future we hope to make the entire collection available in this format.

Figure 1 shows monthly article submission for the 2 year period 2009-2010, showing the dramatic effect of the posting of the impact factor on submissions, which quadrupled from May to September. This has meant that the number of published articles also increased from 12 original research articles in 2009 to 18 in the last months of 2010.

In this final issue of 2010 we publish fifteen original articles of clinical investigation plus seven of basic research.

Souza et al. evaluated the performance of support vector machine, multi-layer perceptron and radial basis function neural networks, as auxiliary tools to identify keratoconus from Orbscan II maps. They conclude that support vector machine, multi-layer percetron classifiers and radial basis function neural network classifiers, trained on Orbscan II data, could represent useful techniques for keratoconus detection.

Parra et al. performed a a detailed histopathological analysis of the open lung biopsy of a series of five cases of ARDS confirmed H1N1. They conclude that viral-like particles can be successfully demonstrated in lung tissue by ultrastructural examination, particularly in those patients without RT-PCR from nasopharingeal aspirate confirmation of the virus. They also found that bronchioles and epithelium, rather than endothelium, are probably the primary target of infection and diffuse alveolar damage the consequence of airways obliteration and dysfunction on innate immunity

Wiedemann et al. correlated preoperative serum Nterminal pro brain type natriuretic peptide with the postoperative outcome and mid term survival rates of coronary artery bypass patients and conclude that these preoperative

Rocha-e-Silva M. Hospital das Clínicas, Faculdade de Medicina, Universidade de São Paulo

Email: mrsilva36@hcnet.usp.br

Tel.: 5511 3069-6235

Copyright (c) 2010 CLINICS - This is an Open Access article distributed under the terms of the Creative Commons Attribution Non-Commercial License (http:// creativecommons.org/licenses/by-nc/3.0/) which permits unrestricted noncommercial use, distribution, and reproduction in any medium, provided the original work is properly cited. of levels the peptide $>502 \mathrm{ng} / \mathrm{ml}$ predict mid-term mortality after isolated coronary artry bypass graft and are associated with significantly higher hospital mortality and perioperative complications.

Franceschini et al. assessed the reliability of the Brazilian version of the Functional Assessment of Cancer TherapyLung combined with the FACT-Lung Symptom Index questionnaires for the measurement of quality of life in patients with lung cancer and conclude that the Brazilian version is reliable, quick and simple to apply, so that this instrument can now be used to properly evaluate the quality of life of Brazilian lung cancer patients.

Jones et al. evaluated the difference in isokinetic strength of hip muscles between patients with knee osteoarthritis vs. matched healthy controls and conclude that patients with osteoarthritis of the knee exhibit lower isokinetic strength in the hip muscles compared with control subjects without osteoarthritis. Strengthening the muscles surrounding the hip joint would appear to have potential to help decrease pain in people with knee osteoarthritis.

Kayıran et al. describe 35 patients with Kawasaki disease in Istambul in the most comprehensive series of children with the disease reported from Turkey. They conclude that adult onset ischemic heart disease may be due to Kawasaki disease in childhood, indicating that further prospective clinical investigations are needed to understand the epidemiology and longterm follow-up of the disease.

Gualco et al. described the clinical pathological characteristics of 1301 cases of pediatric/adolescent lymphomas in patients from different geographic regions of Brazil in what appears to be largest series of pediatric lymphomas presented from Brazil and suggest that some of the results found in this study may reflect heterogeneous socioeconomical status and environmental factors of the Brazilian population in the different regions.

Kikuchi et al. performed a national survey to update hepatocellular carcinoma epidemiology in Brazil and determined clinical and epidemiological profiles of patients with disease in different Brazilian regions. including data from 29 centers on 1,405 patients diagnosed with hepatocellular carcinoma from 2004 to 2009 . They found that the epidemiology, classification, and therapy selection for hepatocellular carcinoma varied among Brazilian regions. Hepatitis $C$ infection was the most common etiology of liver cirrhosis; chemoembolization was the most common therapy employed. Liver cirrhosis was the main risk factor for hepatocellular carcinoma development in Brazil.

Carvalho et al. investigated the clinical and pathological significance of podoplanin expression in the intratumoral stroma and neoplastic cells of early stage uterine cervical cancer. A total of 143 patients with clinical stage I and IIA uterine cervical carcinomas underwent surgery between 2000 and 2007 were surveyed. and conclude that these preliminary results suggest that podoplanin may have a role 


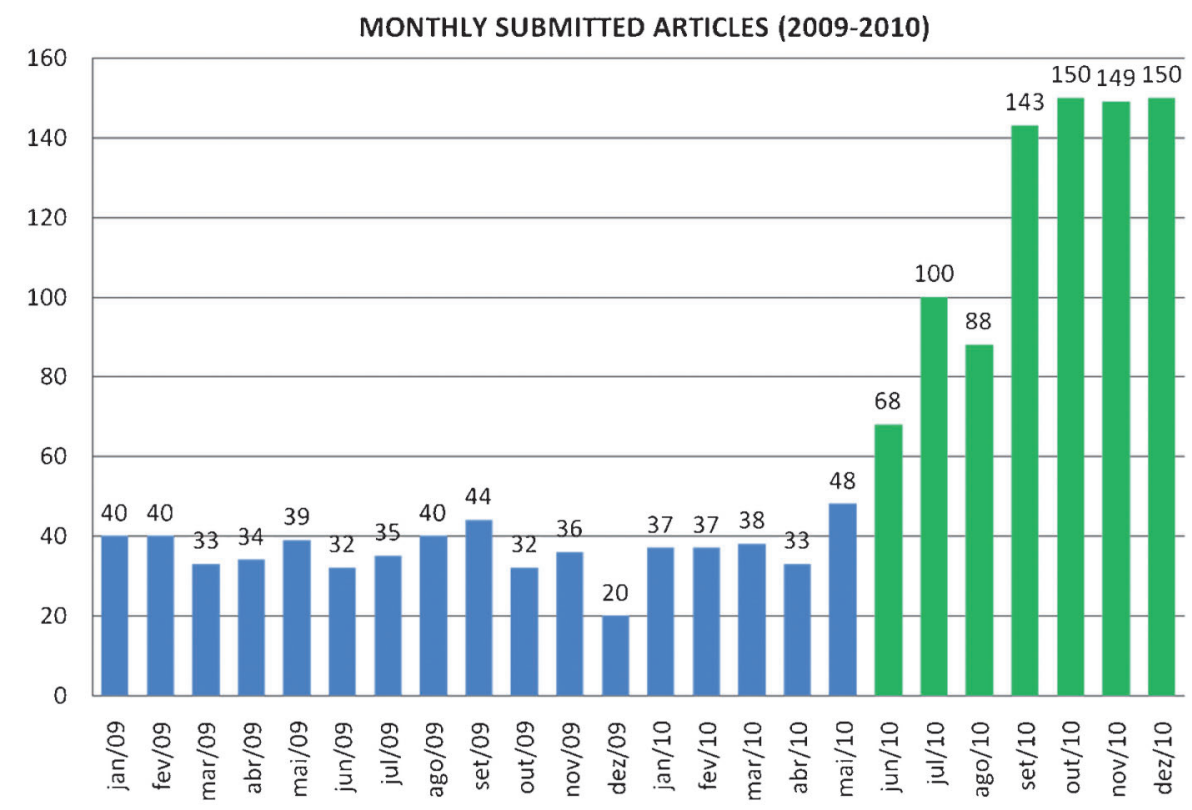

Figure 1 - Monthly submission of articles (all categories) from January 2009 to December 2010, the last figure being an estimate based of the first 15 days of the month.

in host-tumor interactions, and as a result, may represent a favorable prognostic factor for squamous cervical carcinomas

Medeiros et al. determined the occurrence of depression in 25 women who underwent conservative surgery for breast cancer with $(n=25)$ or without $(n=25)$ breast reconstruction and compared them to 25 women without breast cancer. The Beck Depression Inventory was used to measure depression and led to the conclusion that conservative surgery for breast cancer did not affect the occurrence of depression in women, regardless of whether breast reconstruction was performed.

Lopes de Melo et al. evaluate the ability of the forced oscillation technique to detect smoking-induced respiratory alterations, with special emphasis in early alterations; and compared the diagnostic accuracy of forced oscillation technique to spirometric parameters. They conclude that the technique was able to detect the early smoking-induced respiratory involvement when pathologic changes are still potentially reversible. These findings support the use of the forced oscillation technique as a versatile clinic diagnostic tool helping chronic obstructive pulmonary disease prevention, diagnosis and treatment.

Bocalini et al. evaluated the effects of short-term exercise training followed by six weeks of detraining on the functional fitness of older women after a twelve-week water-based exercise program and conclude that the twelveweek water-based exercise program improves the functional fitness parameters as well as quality of life in older women. However, they also found that after a short detraining period of 4 to 6 weeks, the neuromuscular parameters and the quality of life score returned to baseline or to the untrained subject levels.

Morales et al. investigated morphological and hemodynamic patterns of carotid stenoses treated by endarterectomy with patch closure versus stenting through a duplex ultrasound study and claim that both stenting and patch closure were associated with carotid patency and flow restoration. but alert to the fact that the study does not support a general approach to new velocity criteria indiscriminately applied to stented or patched carotids.

Savas et al. investigated the acute effect of phosphodiesterase type 5 inhibitor on erectile dysfunction via evaluating serum oxidative status and prolidase activity of sera of 36 patients with erectile dysfunction and 30 control cases and conclude that the beneficial acute effects of PD5 inhibitor in patients with erectile dysfunction via evaluating serum oxidative status and prolidase activity.

Ramos et al. evaluated the prevalence of ear creases among patients with peripheral arterial disease of the lower limbs, compared to patients without documented atherosclerotic disease and conclude that a higher prevalence of ear creases is independently associated with peripheral arterial disease and may disclose an external marker for risk identification

The seven original articles on themes of Basic Research are as follows.

Achaval et al. investigated the beneficial effects of treadmill training on hindlimb motor function and nerve morphometric parameters in diabetic rats submitted to sciatic nerve crush and conclude that treadmill training is able to accelerate hindlimb motor function recovery in diabetic injured rats and prevent/revert morphometric alterations in proximal nerve portions in non-diabetic and diabetic injured rats.

Valenti et al. evaluated the effects of central catalase inhibition on cardiopulmonary reflex in conscious Wistar rats and conclude that ATZ (3-amino-1,2,4-triazole) injected into the fourth cerebral ventricle increases sympathetic inhibition but does not change the parasympathetic component of the cardiopulmonary reflex in conscious Wistar rats.

Piratelo et al. evaluated the participation of the Angiotensin I, II and 1-7 on the left ventricular hypertrophy of Wistar and Spontaneously Hypertensive rats submitted to sinoaortic denervation. They conclude that not only blood pressure variability and reduced baroreflex sensitivity but also elevated levels of Angiotensin II and reduction 
of the concentration of Angiotensin 1-7 can contribute to the development of left ventricular hypertrophy. They clain that the data indicate that baroreflex dysfunction associated with changes of Renin Angiotensin System may be a predictive factor of left ventricular hypertrophy and cardiac failure.

Ramos et al. compared the Prophylactic and Therapeutic Use of Short-Chain Fatty Acid Enemas in Diversion Colitis in a murine expeimental model. Tey conclude that the therapeutic use of short-chain fatty-acids significantly reduced eosinophilic polymorphonuclear cells numbers in the intestinal wall and in the colonic lumen; it also reversed the atrophy of the colonic mucosa; however prophylactic use did not impede the development of mucosal atrophy.

Kandis et al. studied the effects of Urtica Dioica on Hepatic Ischemia-Reperfusion Injury in Rats and found that liver tissue damage was significantly decreased in treated vs. untreated animals and consequently that it had protective effect on the liver in hepatic ischemia-reperfusion injured rats.

Manhães de Castro et al. investigated the effect of fetal under nutrition on the passive mechanical properties of skeletal muscle of weaned and young adult rats and found that the increase in passive stiffness in skeletal muscle of weaned rat submitted to intrauterine under nutrition it is most likely due to changes in muscle passive stiffness.

Mohd Yusof et al. determine the antiproliferative and apoptosis effects of hot water extract of Chlorella vulgaris on the hepatoma cell line, HepG2 and conclude that it may have anticancer effects possibly by inducing apoptosis signaling cascades via increased expression of P53, Bax and caspase- 3 proteins and reduction of $\mathrm{Bcl}-2$ protein which subsequently lead to increased DNA damage and apoptosis.

We also publish a review on publications dealing the the locomotor system in Brzilian scientific literature, two brief communications and 3 case reports. 